\title{
Méthode pratique d'analyse floristique des pâturages tropicaux (première note)
}

\author{
par J. PAGOT, Z. DERBAL et J. LAHORE
}

\section{1. - GÉNÉRALITÉS}

Le Centre fédéral de Recherches zootechniques est chargé, entre autres choses, de l'étude des pâturages et des techniques prcpres à leur utilisation rationnelle et leur amélioration.

Nos recherches ont commencé en 1951 par l'inventaire des espèces fourragères qui s'est continué en 1952-53. Nous exposons dans ce mémoire les difficultés que nous avons rencontrées dans la détermination des fréquences relatives des différentes espèces utilisables, ainsi que les techniques qui nous ont semblé les plus pratiques et les plus valables pour cette détermination.

\section{2. - HISTORIQUE}

Dès le début des recherches, on a le choix entre des méthodes appréciant soit le nombre des individus de chaque espèce de l'association botanique, soit le rendement en poids de chaque espèce, soit enfin la surface couverte par chaque espèce.

En 1870, Nielsen, au Danemark, employait une technique qui lui permettait d'estimer le nombre d'individus rencontrés dans des échantillons de $0 \mathrm{~m}^{2} \mathrm{ll}$ prélevés dans la prairie à analyser.

Cette méthode ne permet, comme l'indiquent Heden et Lefèvre, de comparer différents pâturages que lorsque les récoltes sont faites à une date physiologique fixe pour l'une des espèces prairiales; elle comporte un grave inconvénient lorsqu'on veut estimer les surfaces occupées par les divers composants de la prairie.

Pour apprécier les surfaces couvertes par les différentes espèces, les méthodes statistiques ont conduit les chercheurs à utiliser des techniques d'échantillonnage au hasard.

Dans la technique du "Point quadrat " (BruceLevy), un peigne de $22 \mathrm{~cm}$. 5, dont les dents sont espacées de $2 \mathrm{~cm}$. 5, est lancé au hasard dans la prairie : on note le nom des plantes qui se trouvent au contact des dents. On multiplie les lancers pour cbtenir un échantillonnage valable.
Dans la technique de De Vries, les échantillons d'une surface de $1 / 4 \mathrm{dm}^{2}$, sont prélevés avec une sonde et l'analyse est faite au laboratoire.

Ces deux techniques donnent avec un certain nombre d'échantillons une idée exacte de la composition de la prairie, mais elles supposent que cette prairie est relativement homogène et que le nombre des espèces est relativement faible.

Dans les pâturages tropicaux, soudanais en particulier, si on trouve dans certaines plaines des peuplements qui peuvent faire penser aux prairies de fauche des zones tempérées, la plupart du temps, on trouve des pâturages du type "savane-brousse ", encombrés d'arbres et de buissons épineux où les espèces herbacées sont très hétérogènes quant à la taille $(0 \mathrm{~m} .15$ à $2 . \mathrm{m} .20)$ (Panicum, Pennisetum).

Les prises d'échantillons par le lancer d'un peigne d'un cercle ou d'un carré de faible surface deviennent très difficiles car l'hétérogénéité de tailles des plantes accompagne une discontinuité du tapis herbeux.

Les surfaces à inventorier étant généralement très grandes le nombre des lancers à faire devient très grand du fait de la technique " au hasard'» et il est difficile de dresser des cartes de repartition en se servant des résultats donnés par l'inventaire au hasard.

Nous exposons ci-après les techniques que nous avons employees. Elles devaient répondre aux caractéristiques suivantes :

- être simples:

- utilisables sur le terrain;

- permettre l'établissement de cartes :

- permettre l'appréciation des pâturages de très grandes surfaces.

\section{3. - DESCRIPTION DES TECHNIQUES}

Nous avons utilisé les méthodes suivantes :

10 Méthode du pied;

20 Méthode du carré:

3o Méthode de Boitel. 
a) Méthode du pied: cette méthode convient pour les pâturages débroussés et les jachères.

On trace dans le pâturage, avec des piquets, des lignes espacées dé 10 à $20 \mathrm{~m}$ on parcourt ces lignes et on procède à l'inventaire des plantes recouvertes par le pied tous les 10 à $20 \mathrm{~m}$. La surface inventoriée à chaque fois représente environ $3 \mathrm{dm}^{2}$.

On doit faire al minimum 25 prises par hectare.

b) Méthode des carrés : cette méthode convient pour tous les pâturages.

On parcourt la zono à inventoricr suivant des lignos à peu près parallèles, espacées de 25 à $50 \mathrm{~m}$, et tous les 25 ou $50 \mathrm{~m}$, on pose sur le sol un cadre de bois de $0 \mathrm{~m} 50 \times 0 \mathrm{~m} 50$.

On identifie et on dénombre toutes les plantes trouvées dans la zone circonscrite par le cadre. L'espacement des cheminements est calculé de façon à obtenir au moins 5 prises par hectare.

Cette methode peut servir à l'établissement de cartes floristiques si on prend la précaution de repérer les points où l'on fait les prises. Ceci est possible $\mathrm{si}$, après ùne reconnaissance rapide du pâturage, on note avec précision les départs et le sens de cheminement.

Par cette méthode, on a étudié également la densité dans les peuplements monospécifiques.

c) Méthode de Boitel : après étude des pâturages du point de vue numérique, nous avons essayé d'utiliser la méthode de Roitel.

Dans la méthode originale, les espèces sont affectées d'une cote variant de 0 à 16 , suivant leur pourcentage relatif. Nous avons utilisé une échelle decimale.

Le pâturage est parcouru de la même façon que dans la méthode précédente, l'appréciation est portée sur le peuplement se trouvant autour de l'observateur dans un cercle de $1 \mathrm{~m} 50$ à $2 \mathrm{~m}$ de rayon.

\section{4. - RÉSULTATS}

Dans les jachères et les prairies naturelles débroussées, nous avons employé la méthode " du pied ».

Un inventaire, fait en août, suivant cette méthode, nous a donné les résultats suivants pour la parcelle ${ }^{\prime \prime} 1$ :

- Cheminements espacés de $20 \mathrm{~m}$ i prises tous les $20 \mathrm{~m}$; 25 prises à l'hectare :

Panicum longijubatum.........

Digitaria sp.................

Setaria pallidifusca...............

Echinochloa colona

Panicum maximum............

Eleusine indica ..............

Eleusine verticillita ............

Paspalum scrobiculatum ........

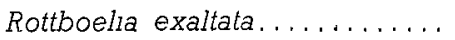

$132 . \ldots 11,96 \%$

$138 \ldots 12,51 \%$

$216 \ldots 18,58 \%$

$90 \ldots 8,15 \%$

3.... $0,27 \%$

$90 \ldots 8,15 \%$

$132 \ldots 11,96 \%$

$81 \ldots 7,34 \%$

$12 . \ldots .1,08 \%$
Crotalaria sp..............

Imperata cylindrica ..........

Ipomea.

Indigo $\operatorname{sp} \ldots \ldots \ldots \ldots \ldots \ldots$

Sida capinifolia .............

Cassia tora ..................

Pennisetum setosum..........

Cymbopogon giganteus .......

Hyparrhenia rufa............

Trifolium sinense $\quad \ldots \ldots \ldots \ldots . .$.

Soit ., $\overline{1.103} \ldots \overline{99,86 \%}$

Dans le pâturage $n^{0} 1$, non débroussé; nous avons opéré suivant les méthodes de Boitel et « des carrés».

Nous avons obtenu les résultats suivants :

(Les chiffres du tableau représentent 20 prises suivant la technique « des carrés » et 20 appréciations suivant celle de Boitel.)

\begin{tabular}{|c|c|c|c|c|}
\hline \multirow{2}{*}{ ESPËCES } & \multicolumn{2}{|c|}{$\begin{array}{l}\text { MÉTHODE } \\
\text { DE BOITEL }\end{array}$} & \multicolumn{2}{|c|}{$\begin{array}{c}\text { MÉTHODE } \\
\text { DES CARRÉS }\end{array}$} \\
\hline & 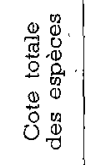 & 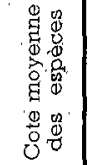 & 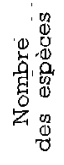 & 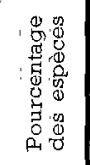 \\
\hline & & $\%$ & & $\%$ \\
\hline Andropogon gajanus. . & 348 & 26,6 & 66 & 7,76 \\
\hline Hyparrhenia rufa...... & 459 & 34,6 & 638 & 75 \\
\hline Hyparrhenia ruprechtil & 71 & 5,5 & 7 & 0,87 \\
\hline Cymbopogon giganteus & 151 & 11,50 & 29 & 3,45 \\
\hline Pennisetum sp........ & 166 & 12,50 & 35 & 4,15 \\
\hline Imperata cylindrica. & 69 & 5,20 & 12 & 1,45 \\
\hline Cassia sp.......... & 10 & 0,1 & 16 & 1,88 \\
\hline Panicum sp. .... & 2 & 0,1 & 一 & - \\
\hline Crotalaria...... & 40 & 3 & 16 & 1,88 \\
\hline Ctenians elegans & 5 & 0,3 & 18 & 2,10 \\
\hline Digitaria $\ldots \ldots \ldots$ & - & - & $1 .-$ & - \\
\hline Michrochloa indica & - & - & 4 & 0,47 \\
\hline Arbustes & - & - & 8 & 0,94 \\
\hline & $1.32 \mathrm{I}$ & 99,40 & 850 & 90,94 \\
\hline
\end{tabular}

On note de grandes différences entre la cote des espèces et le pourcentage obtenu par la méthode " des carrés ». Cette différence s'explique par le fait que dans la méthode' "des carrés" toutes les plantes sont comptées; seul leur nombre intervient, alors que dans la méthode de Boitel l'appréciation est subjective, les espèces reçoivent une cote qui $n$ 'est pas proportionnelle au nombre de pieds, mais l'est plutôt à la surface couverte, et les espèces peu nombreuses reçoivent une cote trop élevée.

Quand le tapis est epais, ce qui se produit en hivernage, les petites espèces, comme les grandes 
au début de leur végétation, échappent à la vue, alors qu'elles sont dénombrées avec la méthode des carrés.

Dans la méthode « des carrés », on obtient les fréquences relatives des espèces présentes sur le pâturage, mais on n'a pas directement de renseignements sur la surface couverte et le volume de la végétation.

Pour ce faire, nous avons commencé l'étude du poids de fourrage fourni par pied et par espèces ainsi que de la densité des peuplements monospécifiques. Ceci fera l'objet d'une note ultérieure.

En comparant les tableaux obtenus avec d'autres parcelles représentant une surface de $420 \mathrm{Ha}$, nous avons remarqué que le rapport des chiffres obtenus pour une même espèce par les deux méthodes et inventorié par le même observateur, variait entre de faibles limites : ainsi pour Andropogon gajanus. le rapport est le suivant :

$$
\frac{\text { cote moyenne en } \%}{\text { fréquence spécifique }}=4
$$

\section{CONCLUSIONS}

Les techniques classiques utilisées pour l'inventaire des prairies de fauche ou d'embouche dans les zones tempérées supposent des conditions qui ne sont qu'exceptionnellement remplies sous les trobiques.

Le principal obstacle à l'emploi des techniques d'échantillonnage par jet de cercles, peignes, etc., réside dans la grande diversité des espèces, la grande variabilité de la taille des individus et l'importance des surfaces dénudées.
La technique des carrés, telle que nous la pratiquons, est une bonne méthode pour l'obtention d'un inventaire floristique exact des pâturages; elle doit être complétée par une étude quantitative permettant d'apprécier le rendement du pâturage.

Quand un observateur a acquis, par la pratique de la méthode "des carrés 》 dans une zone donnée, une expérience suffisante, il peut alors utiliser pour lappréciation des grandes surfaces, la méthode de Boitel; les résultats qu'il obtiendra devront être acceptés avec toutes les réserves que comportent les méthodes d'appréciation subjective.

Centre fédéral de Recherches zootechniques de Sotuha (Soudan) A.O.F.

\section{BIBLIOGRAPHIE}

ESSOCLATION FRANÇAISE DE ZOOTECHNIE. C. R. Jonrnées d'études sur l'alimentation à la prairie, Paris, 3-4-5 décembre 1951.

BIEICHERT (H.), - Exfolgreiche Grundlandwirtschaft. Die Deutsche Landwirtscháft, Heft 3, 150.

BCEUF (F.) et VEYSSEREAU (A.). - Recherche et expérimentation en Agriculture. 1, 2, 1948. J.-B. Baillière et Fils, édit. Paris.

GFCHON (L.). - Observations sur l'amélioration des prairies de fauche. Bull. Techn. Inlorm. Mg. Serv. Agr. 75 (1952), 762-763.

HAVARD DUCLOS (R.). - Pâturages tropicaux. Fasc. 1 (1952). Libr. Agric. Horti. Forest. Ménagère, 26, rue Jacob, Paris-6o.

HEDIN ( $L_{\text {. }}$ ) et LEFEBVRE. - Les méthodes d'analyse botanique dans l'étude agronomique des prairies. Ann. I.N.R.A.-B (1951), 376,407. 